\title{
Correction: Chaperonin 60 sustains osteoblast autophagy and counteracts glucocorticoid aggravation of osteoporosis by chaperoning RPTOR
}

Wei-Shiung Lian ${ }^{1,2}$, Jih-Yang Ko ${ }^{3}$, Yu-Shan Chen ${ }^{1,2}$, Huei-Ching Ke ${ }^{1,2}$, Shin-Long Wu ${ }^{1,2}$, Chung-Wen Kuo ${ }^{1,2}$ and Feng-Sheng Wang ${ }^{1,2,4}$

\section{Correction to: Cell Death \& Disease https://doi.org/10.1038/s41419-018-0970-6, published online 17 September 2018}

Following publication of this article, the authors realized that there were 1) errors made in the author affiliations and that 2) a typo in a grant number needed to be corrected. The corrected author affiliations and grant numbers are listed below. We apologize for the inconvenience.

1) Wei-Shiung Lian ${ }^{1,2}$, Jih-Yang $\mathrm{Ko}^{3}$, Yu-Shan $\mathrm{Chen}^{1,2}$, Huei-Ching $\mathrm{Ke}^{1,2}$, Shin-Long $\mathrm{Wu}^{1,2}$, Chung-Wen Kuo ${ }^{1,2}$, Feng-Sheng Wang ${ }^{1,2,4}$

1 Core Laboratory for Phenomics and Diagnostic, Kaohsiung Chang Gung Memorial Hospital, Kaohsiung, Taiwan
2 Department of Medical Research, Kaohsiung Chang Gung Memorial Hospital, Kaohsiung, Taiwan

3 Department of Orthopedic Surgery, Kaohsiung Chang Gung Memorial Hospital, Kaohsiung, Taiwan

4 Graduate Institute of Clinical Medical Science, Chang Gung University College of Medicine, Kaohsiung, Taiwan

2) In the Acknowledgements section, grant numberCMRPG8H00112 is incorrect. The correct grant number is CMRPG8H0111.

This has been corrected in both the PDF and HTML versions of the Article.

Published online: 03 October 2019

\footnotetext{
Correspondence: Feng-Sheng Wang (wangfs@ms33.hinet.net)

${ }^{1}$ Core Laboratory for Phenomics and Diagnostic, Kaohsiung Chang Gung

Memorial Hospital, Kaohsiung, Taiwan

2Department of Medical Research, Kaohsiung Chang Gung Memorial Hospital,

Kaohsiung, Taiwan

${ }^{3}$ Department of Orthopedic Surgery, Kaohsiung Chang Gung Memorial

Hospital, Kaohsiung, Taiwan

${ }^{4}$ Graduate Institute of Clinical Medical Science, Chang Gung University College

of Medicine, Kaohsiung, Taiwan
}

(c) (i) Open Access This article is licensed under a Creative Commons Attribution 4.0 International License, which permits use, sharing, adaptation, distribution and reproduction (1) in any medium or format, as long as you give appropriate credit to the original author(s) and the source, provide a link to the Creative Commons license, and indicate if changes were made. The images or other third party material in this article are included in the article's Creative Commons license, unless indicated otherwise in a credit line to the material. If material is not included in the article's Creative Commons license and your intended use is not permitted by statutory regulation or exceeds the permitted use, you will need to obtain permission directly from the copyright holder. To view a copy of this license, visit http://creativecommons.org/licenses/by/4.0/. 